\title{
Contents, Vol. 53, 1924
}

\section{lnhalts-Verzeichnis.}

Eigenarbeiten. seite

Beck, Oscar, Zur Pathologie der Nebenhöhlen der Nase

bei der rhinogenen, retrobulbären Neuritis optica . 295 Bergmeister, B., Über nekrotisierende intraokulare Tuber-

kulose und Tuberkulose des Sehnerven • 175

Birch-Hirschfeld, P., Weitere Erfahrungen über Behandlung

infektiöser Hornhauterkrankungen mit ultraviolettem

Lichte. .. 151

-, Zur Schutzbrillenfrage 7

Blatt, Nicolaus, Einseitige kongenitale Anomalie des Mus-

culus orbicularis am linken Auge 87

Distichiasis congenita vera 325

Bliedimg, C, Beitrag zur Behandlung der Stauungspapille

durch Balkenstich 199

Deutschmann, B., Gibt es eine operative Beeinflussung der

Sehnervenatrophie?

Friede, B., Epiphora, bedingt durch funktionelles Ektro-

pium $\mathrm{b}$ eider Unterlider $\quad 95$

Gernet, B. von, Zur Tarsusexzision 211

Grimminger, Walther, Über Blutimbibition der vorderen

Linsenkapsel 339

- $\quad$ - Ein Beitrag zur Entstehung des ,traumatischen

Sehnervenscheiden-Hämatoms"

41

Hamburg, J.,Über Parazenthesekuren bei chronischer Irido-

cyclitis 55

Hamburger, C, Glaukombehandlung. Berichtigung ...

Hoeve, J. van der, Vererbbarkeit nach Keratokonus. . . 342Knüsel, 0., Vitale Färbungen am menschlichen Auge. IVa.

Lymphgefäßstudien an der Augenbindehaut ...

- $\quad$ - IV b. Lymphgefäßstudien an der Augenbindehaut 191

Krebs, H, siehe Bömer, P.

Kumer, L. und L. Sallmann, Zur Radiumtherapie des

Trachoms 23

Lindner, K., Über das Verhältnis der Erreger eitriger Binde-

hautentzündungen zur Hornhaut 157

Über die Infektionsgefahr nach bulbuseröffnenden

Operationen 305

Bömer, P. und H. Krebs, Beitrag zur Hamburger schen 
Glaukomtherapie $\quad 13$

Sachs, if., Über Schielen und Stottern 285

Sallmann, L., siehe Kumer, L.

Stajduhar, J., Über einen Fall von Ichthyosis und Binde-

hauttuberkulose .... 321

$-\mathrm{IV}-$

Seit $\theta$

Stoewer, P., Ein Fall von ophthalmolplegischer Migräne . 207Wick, W.\} Muskelfaltung bei

Schieloperationen .... 203 Plastische Bildung des Bindehautsackes .... 83

Aus der Praxis fur die Praxis.

Gilbert, W., Zur Jodbehandlung der Cataract ....

- - Zur operativen Behandlung der Optikusatrophie 344

Hofe, Karl vom. Die Bonner Lesetafel

Ollendorf, A., Die operative Behandlung des sekundären

Auswärtsschielens

345

Berichte über die deutsche ophthalmologische Literatur.

Diagnostik und Extraktion von Fremdkörpern (1923).

Von San.-Rat Dr. Ed. Asmus in Düsseldorf .... 101 Untersuchungsmethoden I. und II. Semester 1923. Von

San.-Rat Dr. R. Kramer in Wien $\quad .215$

Spezielle Pathologie und Therapie im Jahre 1923. Von

San.-Rat Dr. Konrad Schrader in Gera 351

Gesellschaftsberichte.

Heidelberg $1924 \quad .223$

Bericht über die 44.Versammlungder Ophthalmologischen

Gesellschaft in Heidelberg am 12. bis 14. Juni 1924225 Bericht über die fünfte Sitzung der

Ophthalmologischen

Abteilung der Gesellschaft für Wissenschaft und

Leben im rhein.-westf. Industriebezirk am 19. I. 1924

in Essen. Von Dr. R. Heßberg in Essen Ill

Berliner Augenärztliche Gesellschaft. Sitzungen vom 24.1.,

21. II. und 27. III. 1924123

Ophthalmologische Gesellschaft in Wien. Sitzungen vom

10. XII. 1923, 21. 1.1924132

Sitzungen vom 18. II. und 3. III. 1924265

Verein der Augenärzte von Ost- und Westpreußen. Sitzung

vom 21. VII. 1923278

Ungarische Ophthalmologische Gesellschaft. Sitzungen

vom 7. X., 4. XL und 2. XII. 1923137

Sitzung vom 6. I. und 3. II. 1924280

Diagnose und Therapie 143, 434

Buchbesprechungen 146, 283, 440

Personalien und Tagesnachrichten 150, 284, 440

Mitteilung der Schriftleitung 284

Druckfehlerberichtigung 284

S. Sachregister441 
Namenregister 451 